\title{
Generation of Terahertz Radiation by a Photoconductive Antenna
}

\author{
V.N. Trukhin ${ }^{a, *}$, A.V. Andrianov ${ }^{a}$ AND N.N. ZINOV'EV ${ }^{a, b}$ \\ ${ }^{a}$ Ioffe Physico-Technical Institute \\ Polytekhnicheskaya 26, St. Petersburg, 194021, Russia \\ ${ }^{b}$ Department of Physics, University of Durham, Durham DH1 3LE, UK \\ We report on the first principle calculations of photocarriers kinetics \\ in a photoconductive antenna excited by an ultrashort optical laser pulse. \\ The solution of non-equilibrium Boltzmann equation is used to derive the \\ expression for the irradiated electric field. The analysis reveals the important \\ role of non-uniform photocarrier distribution inside the active layer in the \\ formation of the terahertz radiation from the emitter in both collinear and \\ anti-collinear directions.
}

PACS numbers: 42.65.Ky, 41.60.-m, 78.47.--p

\section{Introduction}

The time-domain coherent $\mathrm{THz}$ spectroscopy of condensed matter has got considerable attention during the last decade due to great perspectives of its applications in many areas of science and technology. A critical component of the coherent $\mathrm{THz}$ spectrometer is a $\mathrm{THz}$ emitter. Up to now the most popular $\mathrm{THz}$ emitters are semiconductor based photoconductive antennas excited by ultrashort pulses of visible or near-infrared lasers. The physical principle of such emitter employs the short current pulse in biased semiconductor with small lifetime of nonequilibrium carriers created by the laser pulse [1]. The current pulse radiates the $\mathrm{THz}$ electromagnetic waves. The large-aperture photoconductive antennas were studied experimentally and theoretically in many papers [2-4]. However, in the most of the published works the analysis of the $\mathrm{THz}$ emission was performed in frame of simplified approaches which did not take into account the propagation of a short pump pulse through a semiconductor etc. We have developed a model describing the emitted $\mathrm{THz}$ field based on the solution of the Boltzman semiconductor and Maxwell equations for a photoconductive THz emitter and demonstrate the application of this model for low-temperature (LT)-GaAs photoconductive antennas.

*corresponding author; e-mail: valera.truchin@mail.ioffe.ru 


\section{Calculation of $\mathrm{THz}$ emission field for large-aperture emitters}

Let us consider a large-aperture photoconductive emitter excited by a short light pulse with the energy of quantum exceeding the band energy of the material. We will assume that the excitation is uniform and propagates in $y$-direction. Also we will suppose that the bias field is uniform too, directed in $z$-directed and is considerably stronger than the electric field of $\mathrm{THz}$ radiation. The symmetry of the task allows one to consider the set of Maxwell equations for $\mathrm{THz}$ radiation fields $\boldsymbol{E}(\boldsymbol{r}, t)$ and $\boldsymbol{H}(\boldsymbol{r}, t)$ in one-dimensional representation. After Fourier transforms we will get the following wave equation for the Fourier component of electric field:

$$
\frac{\mathrm{d}^{2} E_{z}^{\omega}}{\mathrm{d} y^{2}}+\frac{\omega^{2}}{c^{2}} \varepsilon E_{z}^{\omega}=-\mathrm{i} \omega \mu_{0} J_{z}^{\omega}
$$

where $\varepsilon, \mu_{0}$ have usual meaning, $c$ and $\omega$ are light velocity in vacuum and frequency of the $\mathrm{THz}$ radiation, $J_{z}^{\omega}$ is the Fourier component of the density of photoinduced current. The photocurrent density can be calculated from the nonequilibrium distribution function $f_{\mathrm{e}, \mathrm{h}}$ of free carriers

$$
J_{z}(t, y)=\frac{\hbar e}{4 \pi^{3}} \int k_{z}\left[f_{\mathrm{e}}(\boldsymbol{k}, t, \boldsymbol{r}) / m_{\mathrm{e}}-f_{\mathrm{h}}(\boldsymbol{k}, t, \boldsymbol{r}) / m_{\mathrm{h}}\right] \mathrm{d} \boldsymbol{k}
$$

where $\boldsymbol{k}$ is the electron wave vector, $m_{\mathrm{e}}$ and $m_{\mathrm{h}}$ are the effective masses of free electrons and holes, respectively. From (2) it is clear that in approximation of $m_{\mathrm{e}} \ll m_{\mathrm{h}}$ we may omit the second term in (2). The holes are assumed to form a homogeneous background for recombining electrons. Omitting index e of $f_{\mathrm{e}}$ for convenience, the distribution functions of electrons is found from the Boltzmann equation $[5,6]$ :

$$
\frac{\partial f}{\partial t}+\frac{\hbar \boldsymbol{k}}{m} \frac{\partial f}{\partial \boldsymbol{r}}+\frac{e}{\hbar} \boldsymbol{E} \frac{\partial f}{\partial \boldsymbol{k}}=-\Gamma(f-\tilde{f})+\gamma f+G(t, y) \frac{\pi^{2} \delta\left(k-k_{0}\right)}{k_{0}^{2}},
$$

where $\Gamma$ is the inverse time of the momentum relaxation, $\gamma$ is the inverse lifetime of the nonequilibrium electrons in the conduction band, $k_{0}^{2}=2\left(\hbar \omega_{0}-E_{\mathrm{g}}\right)$ $m_{\mathrm{e}} m_{\mathrm{h}} /\left[\hbar^{2}\left(m_{\mathrm{e}}+m_{\mathrm{h}}\right)\right]$ is the module of the wave vector of photoexcited electrons, $E_{\mathrm{g}}$ is the forbidden gap, $\hbar \omega_{0}$ is the quantum energy of pump. The pump generation rate $G(y, t)$ is

$$
G(t, y)=\frac{\alpha(1-R) I_{0}}{\hbar \omega_{0}} \exp (-\alpha y) \exp \left(-\left(t-\frac{y}{\nu_{0}}\right)^{2} / 2 \Delta^{2}\right)
$$

where $I_{0}, \alpha, R$ are the pump intensity, absorption coefficient and reflection coefficient of semiconducting layer at the frequency of pump, $\nu_{0}$ is the group velocity of pump, $\Delta$ is duration of pump pulse. Equation (4) takes into account finite absorption of the external pump pulse source in the layer that leads to the nonuniform current density along $y$-axis. The Fourier component of the current density, $J_{z}^{\omega}$, obtained from (2) and (3), can be written as 


$$
J_{z}^{\omega}=\frac{e^{2}}{m_{e}} E_{0}\left[\gamma\left(k_{0}\right)-\mathrm{i} \omega\right]^{-1}\left[\Gamma\left(k_{0}\right)+\gamma\left(k_{0}\right)-\mathrm{i} \omega\right]^{-1} G \omega(\omega, y),
$$

where

$$
G_{\omega}(\omega, y)=\frac{\alpha(1-R) I_{0} \Delta}{\hbar \omega_{0}} \exp (-\alpha y) \exp \left(-\frac{\omega^{2} \Delta^{2}}{2}\right) \exp \left(\mathrm{i} \omega \frac{y}{\nu_{0}}\right) .
$$

The external electric field at frequency $\omega$ is the sum of two terms, the solutions of homogeneous and inhomogeneous wave equation. In the case of $\alpha d>1$ using the boundary continuity conditions for $\boldsymbol{E}$ and $\boldsymbol{H}$ vectors finally we obtain for the $\mathrm{THz}$ electric field at output and input surfaces of the emitter, respectively:

$$
\begin{gathered}
E_{\omega}(\omega, d+)=-\left(\frac{\mu_{0}}{\varepsilon_{0}}\right)^{1 / 2} A(\omega)\left(1-\mathrm{i} \frac{\omega N}{\alpha c}\right)^{-1}\left(1+\mathrm{i} \frac{\omega \delta n}{\alpha c}\right)^{-1} \\
\times \frac{\left(1-\mathrm{i} \frac{\omega\left(n_{0}+n_{\mathrm{c}}\right)}{\alpha c}\right) 2 n}{\left(n+n_{\mathrm{c}}\right)^{2}} \exp \left(\mathrm{i} \frac{\omega}{c} n d\right)\left[1-r^{2} \exp \left(\mathrm{i} \frac{\omega}{c} 2 n d\right)\right]^{-1}, \\
E_{\omega}(\omega, 0-)=-\left(\frac{\mu_{0}}{\varepsilon_{0}}\right)^{1 / 2} A(\omega)\left[1-r^{2} \exp \left(\mathrm{i} \frac{\omega}{c} 2 n d\right)\right]^{-1} \\
\times\left[\frac{\left(1-\mathrm{i} \frac{\omega N}{\alpha c}\right)^{-1}}{n+n_{\mathrm{c}}}+\frac{\left(1+\mathrm{i} \frac{\omega \delta n}{\alpha c}\right)^{-1} r}{n+n_{\mathrm{c}}} \exp \left(\mathrm{i} \frac{\omega}{c} 2 n d\right)\right]
\end{gathered}
$$

where $n_{0}=c / \nu_{0}, n=\sqrt{\varepsilon}, n_{\mathrm{c}}=\sqrt{\varepsilon_{\mathrm{c}}}$ - are refractive indices of the material and surroundings, respectively,

$$
\begin{aligned}
& \tau^{-1}=\gamma, \tau_{\mathrm{p}}^{-1}=\Gamma+\gamma, \delta n=n-n_{0}, N=n+n_{0}, r=\left(n-n_{\mathrm{c}}\right) /\left(n+n_{\mathrm{c}}\right), \\
& A=\frac{e^{2}}{m_{\mathrm{e}}} E_{0} \frac{(1-R) I_{0} \Delta}{\hbar \omega_{0}} \tau \tau_{\mathrm{p}} \exp \left(-\frac{\omega^{2} \Delta^{2}}{2}\right)(1-\mathrm{i} \omega \tau)^{-1}\left(1-\mathrm{i} \omega \tau_{\mathrm{p}}\right)^{-1} .
\end{aligned}
$$

The wave form of the electric field in a far field zone is calculated using diffraction integral [7]. Figures 1 and 2 show the wave forms calculated for forward and

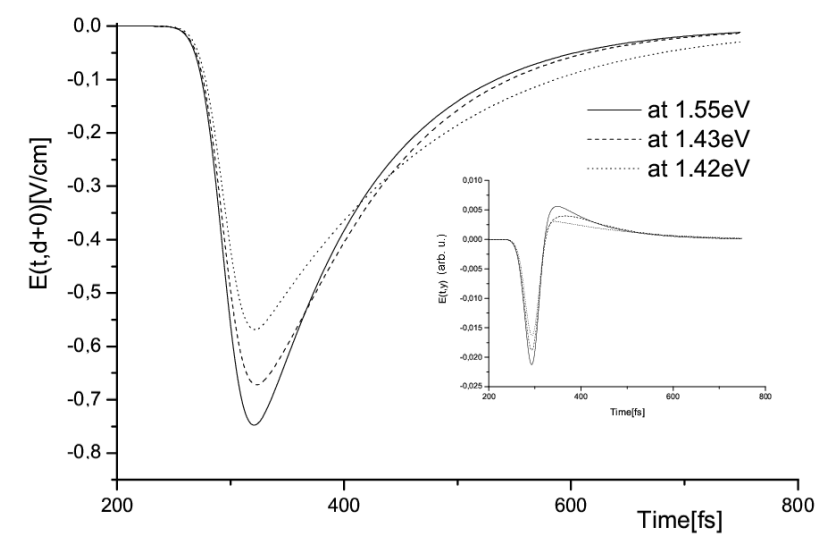

Fig. 1. Theoretical wave forms forwardly emitted from the slab of LT-GaAs . The inset shows these wave forms in far field zone. Pulse duration is $15 \mathrm{fs}, I_{0}=1 \mathrm{MW} / \mathrm{cm}^{2}$. The parameters: $\tau=100 \mathrm{fs}, \tau_{\mathrm{p}}=70 \mathrm{fs}, n=3.5, n_{0}=3.65$ are taken from literature. 


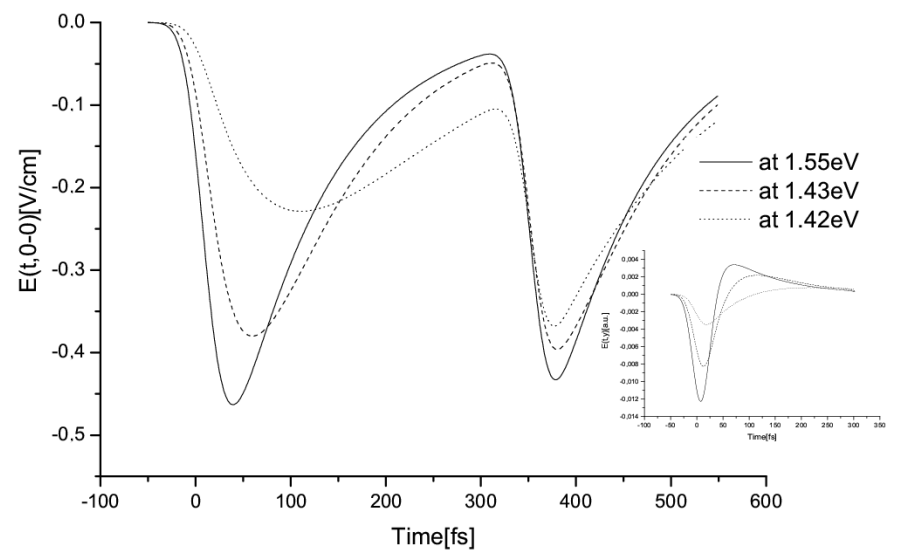

Fig. 2. Theoretical wave forms backwardly emitted from the slab of LT-GaAs. The inset shows these wave forms in far field zone. The same data as in Fig. 1 was used.

backward emitted $\mathrm{THz}$ radiation from a semiconductor emitter. It is seen that the details, the spectral content and the field amplitude of the wave forms, strongly depend on the choice of pump quantum energy $\hbar \omega_{0}$. For the backward emitted $\mathrm{THz}$ field, the spectral content of the $\mathrm{THz}$ wave forms is very sensitive to the sum of the time of flight of the pump and time of flight of the $\mathrm{THz}$ pulse on the scale of $\alpha^{-1}$. For the forward emitted $\mathrm{THz}$ pulse the important parameter is the difference of the time of flight of the pump and time of flight of the $\mathrm{THz}$ pulse on the scale of $\alpha^{-1}$. The generation mechanism is, therefore, the composite product of two localized sources of $\mathrm{THz}$ radiation. If $a d>1$, the generation is restricted to the input boundary of the emitter, whereas at $a d<1$, the generation also occurs at the output boundary. Such scenario, to some extent, is similar to the mechanism of $\mathrm{THz}$ generation at nonlinear wave conversion discussed in [8].

\section{References}

[1] D.H. Auston, Appl. Phys. Lett. 26, 101 (1975).

[2] J.T. Darrow, X.C. Zhang, D.H. Auston, J.D. Morse, IEEE J. Quantum Electron. 28, 1607 (1992).

[3] P.K. Benicewicz, J.P. Roberts, A.J. Taylor, J. Opt. Soc. Am. B11, 2533 (1994).

[4] L. Hou, X. fang Sun, W. Shi, in: Proc. 2006 Joint 31st Int. Conf. on IMMW and 14 th Int. Conf. on THz Electronics, Eds. Xue Chu Shen, Wei Lu, Jie Zhang, Wen Bin Dou, IEEE Inc. 2006, p. 119.

[5] V.I. Belinicher, S.M. Ryvkin, Zh. Eksp. Teor. Fiz. 81, 353 (1981).

[6] H.J. Stocker, H. Kaplan, Phys. Rev. 150, 619 (1966).

[7] M. Born, E. Wolf, Principles of Optics, Pergamon, New York 1970.

[8] N.N. Zinov'ev, A.S. Nikoghosyan, J.M. Chamberlain, Phys. Rev. Lett. 98, 044801 (2007). 